\title{
Imported chikungunya cases in an area newly colonised by Aedes albopictus: mathematical assessment of the best public health strategy
}

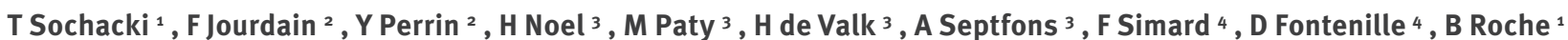

1. UMI IRD/UPMC Unité de Modélisation Mathématique et Informatique des Sytèmes Complexes (UMMISCO), Bondy, France

2. Centre National d'Expertise des Vecteurs (CNEV), Montpellier, France

3. Institut National de Veille Sanitaire, Paris, France

4. UMR CNRS/IRD/UM Maladies Infectieuses et Vecteurs: Ecologie, Génétique Evolution et Contrôle (MIVEGEC), Montpellier, France

Correspondence: Benjamin Roche (benjamin.roche@ird.fr)

Citation style for this article:

Sochacki T, Jourdain F, Perrin Y, Noel H, Paty M, de Valk H, Septfons A, Simard F, Fontenille D, Roche B. Imported chikungunya cases in an area newly colonised by Aedes albopictus: mathematical assessment of the best public health strategy. Euro Surveill. 2016;21(18):pii=30221. DOI: http://dx.doi.org/10.2807/1560-7917. ES.2016.21.18.30221

Article submitted on 21 April 2015 / accepted on 04 November 2015 / published on 05 May 2016

We aimed to identify the optimal strategy that should be used by public health authorities against transmission of chikungunya virus in mainland France. The theoretical model we developed, which mimics the current surveillance system, predicted that without vector control (VC), the probability of local transmission after introduction of viraemic patients was around $2 \%$, and the number of autochthonous cases between five and 15 persons per hectare, depending on the number of imported cases. Compared with this baseline, we considered different strategies (VC after clinical suspicion of a case or after laboratory confirmation, for imported or autochthonous cases): Awaiting laboratory confirmation for suspected imported cases to implement VC had no significant impact on the epidemiological outcomes analysed, mainly because of the delay before entering into the surveillance system. However, waiting for laboratory confirmation of autochthonous cases before implementing VC resulted in more frequent outbreaks. After analysing the economic cost of such strategies, our study suggested implementing VC immediately after the notification of a suspected autochthonous case as the most efficient strategy in settings where local transmission has been proven. Nevertheless, we identified that decreasing reporting time for imported cases should remain a priority.

\section{Introduction}

Environmental changes are a significant cause for concern for public health authorities $[1,2]$. Global warming [3], decline in biodiversity [4], urbanisation [5] or globalisation [6] can facilitate the spread of vectors and increase pathogen transmission. While some of these changes occur over the course of several years or decades and could therefore represent a problem in the long term, invasive vector species that can transmit pathogens such as dengue or chikungunya viruses expose new human populations to new pathogens already today [7-10].

This risk of disease transmission by invasive vectors was particularly significant in 2014. Indeed, the occurrence of a large chikungunya outbreak on several Caribbean islands [11-13] during the season of Aedes albopictus vector activity in southern France [14] became problematic for public health risk management because of the high number of imported cases recorded [15]. While virus transmission remained under control in 2014, with moderate local transmission of dengue and chikungunya viruses [16], this situation can be expected to repeat in the future and requires an efficient public health strategy rooted in quantitative risk assessment under the current and alternative strategies.

For chikungunya virus, the current strategy in vectorinfested areas and during the season of vector activity comprises the following components [15]: Suspected cases (defined by the presence of acute fever and joint pains not explained by another medical condition) are immediately reported to public health authorities. Such cases are then confirmed by serology (IgM-positive or a fourfold increase in IgG titre) or detection of viral nucleic acids in plasma by real-time reverse-transcription PCR (RT-PCR).

If the suspected case is imported from a chikungunyaendemic or -epidemic area, vector control (VC) measures, such as spraying adulticides to temporarily reduce the density of adult mosquitoes and removing stagnant water to decrease the number of breeding sites, are implemented within $200 \mathrm{~m}$ of the places in mainland 
Structure of the mathematical model assessing public health strategies again chikungunya virus transmission, mainland France

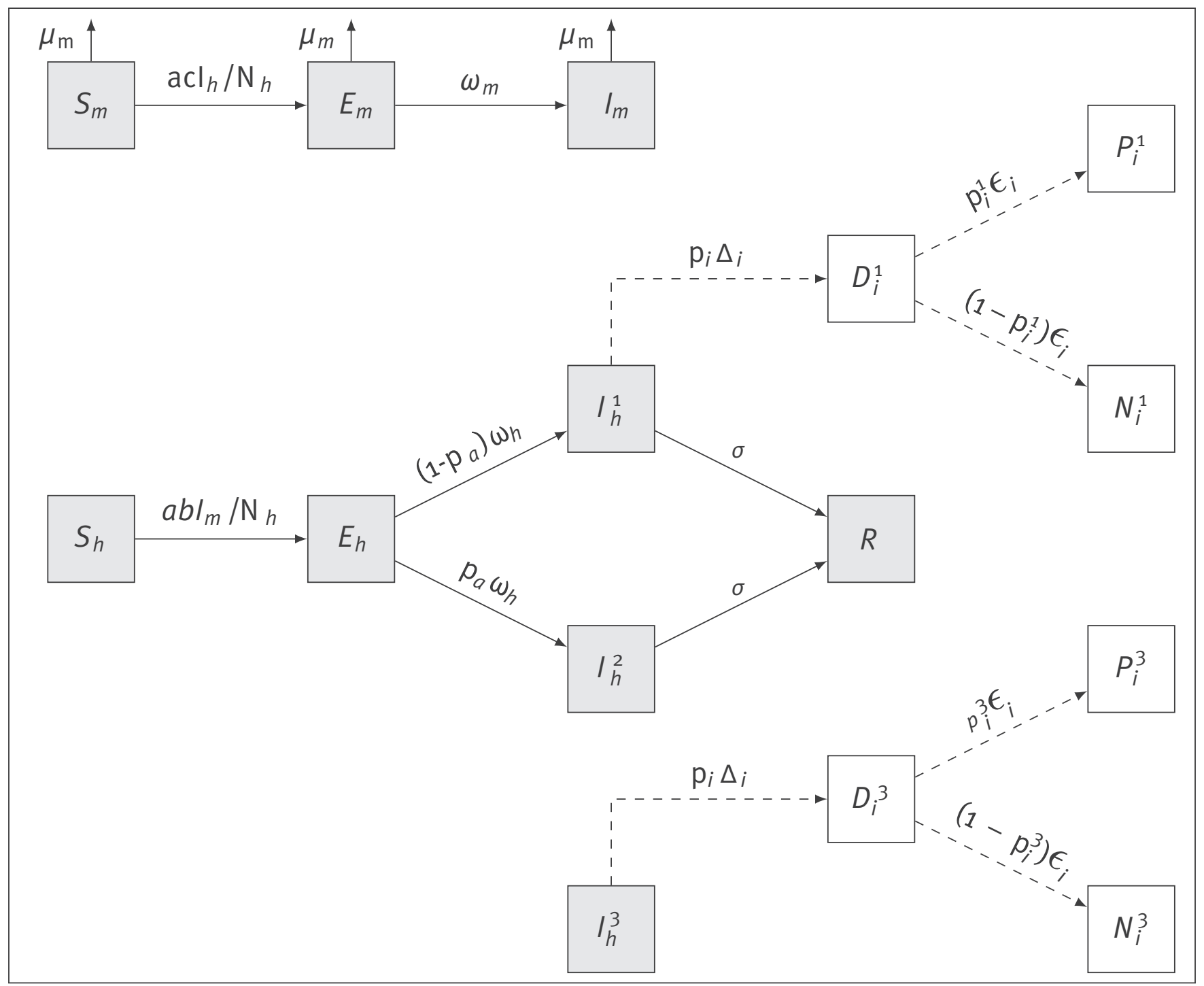

France visited by the patient during the likely viraemic period (from the day before until seven days after the onset of symptoms), without waiting for laboratory confirmation. In the absence of proof of local transmission (i.e. without any previous biological confirmation of an autochthonous case), VC measures around suspected autochthonous cases are delayed until these cases are laboratory-confirmed.

The notification of a laboratory-confirmed autochthonous case triggers immediate epidemiological and entomological investigations and control measures, including active case finding in the neighbourhood of the case's residence and in other areas visited by the case. VC is then implemented around the confirmed cases and around any further suspected autochthonous cases.

One of the main questions is whether or not waiting for laboratory confirmation is relevant before implementing VC locally. Intuitively, the most efficient strategy in epidemiological terms would be to implement VC as soon as possible, i.e. just after the notification of a clinically suspected case, whether the case is imported or locally acquired (autochthonous). However, the number of false notifications (clinical suspicion of chikungunya cases where chikungunya virus is not involved) is substantial because of the low specificity of the symptoms: in 2015 , only six cases were confirmed among 532 notified cases (personal communication: Alexandra Septfons, INVS, April 2015). Therefore, the efficiency of such strategies has to be assessed. In order to design the most efficient public health strategy, it is important to quantify how many secondary cases occur because VC is delayed until laboratory confirmation for the suspected imported and autochthonous cases.

We designed a mathematical model for chikungunya control, which could be easily extended to other 


\section{FIGURE 2}

Modelled probability of epidemics (defined as simulation with at least five local cases) and number of autochthonous cases (in simulations identified as epidemic) in simulations without vector control

Montpellier
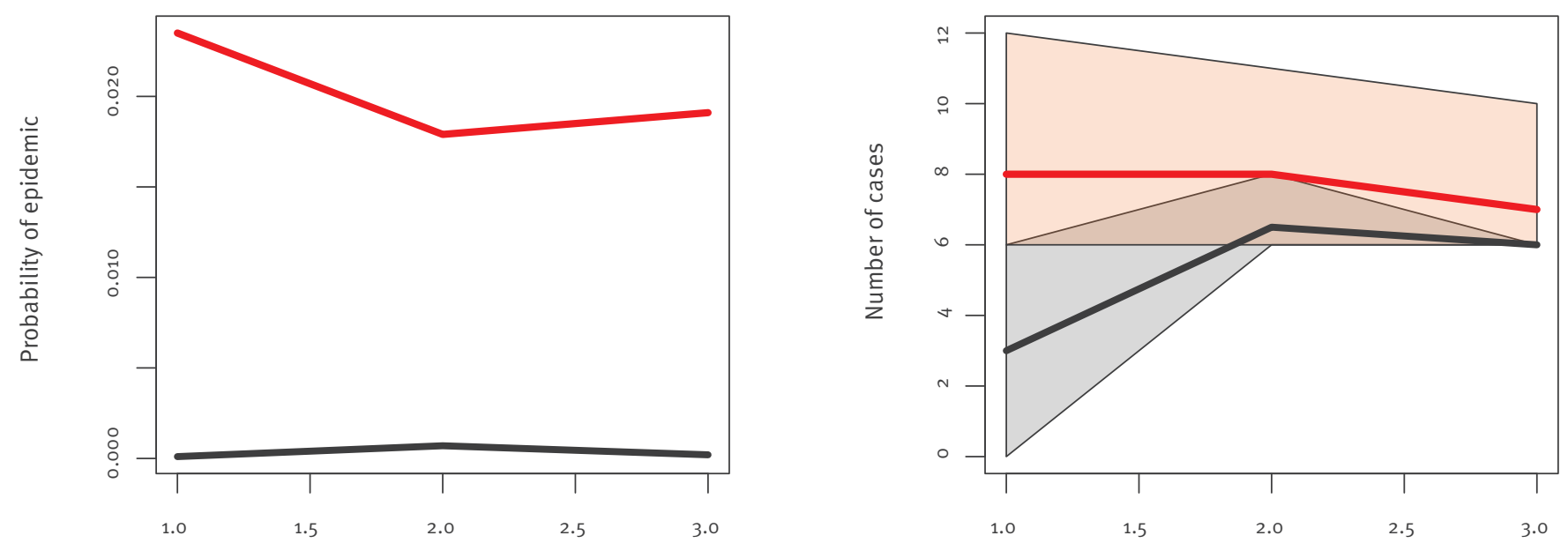

Lyon

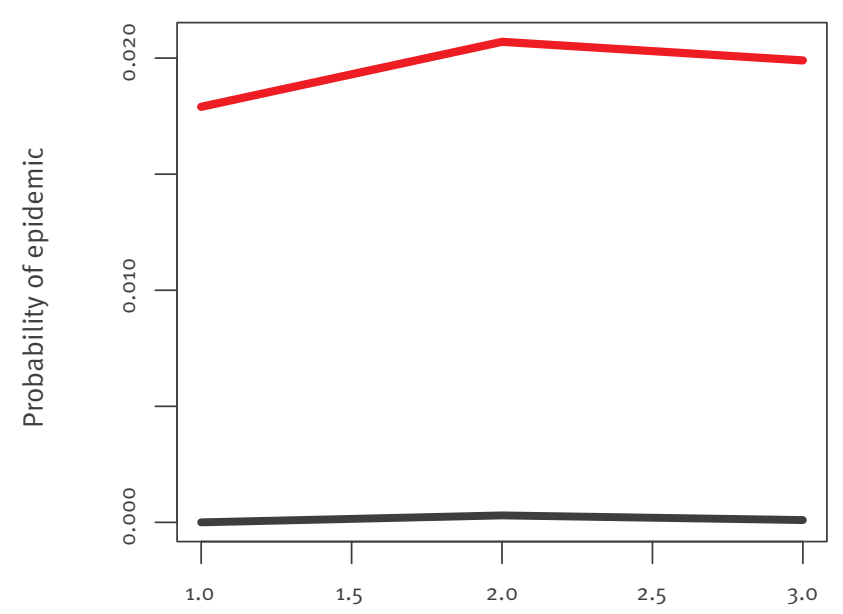

Number of imported cases

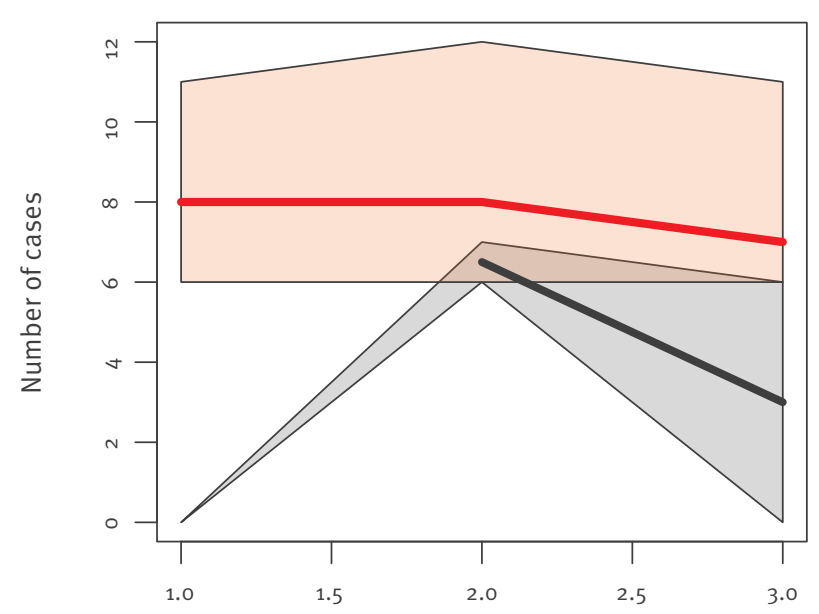

Number of imported cases

Red lines: maximum mosquito density ( 800 females/ha); black lines: minimum density ( 20 females/ha). The confidence intervals are the $95 \%$ interval over the 10,000 simulations defined by the 2.5 th and 97.5 th percentiles.

vector-borne diseases (e.g. dengue or Zika virus infections) and/or surveillance systems, in order to analyse theoretically four possible public health strategies (VC after clinical suspicion or after laboratory confirmation, for imported or autochthonous cases). We include in this model the surveillance data obtained during the 2014 season, suggest what could be the optimal strategy for the following years and make recommendations how to improve the surveillance system.

\section{Methods}

We developed a stochastic epidemiological model [17] rooted within the SIR (susceptible-infectious-recovered) framework where populations of humans and vectors are compartmentalised according to their infection status (Figure 1). Human individuals start simulation in a susceptible state $\left(\mathrm{S}_{\mathrm{h}}\right)$ and then can be infected through bites of infectious vectors $\left(\mathrm{I}_{\mathrm{m}}\right)$ at rate $a$ and with the probability $b$. When infected $\left(\mathrm{E}_{h}\right)$, these individuals become, at a rate $\omega_{h}$, infectious and symptomatic $\left(\mathrm{I}_{\mathrm{h}}{ }^{1}\right)$ with the probability $\left(1-p_{a}\right)$, or asymptomatic $\left(\mathrm{I}_{\mathrm{h}}{ }^{2}\right)$ with the probability $p_{a}$. Finally, infectious individuals 


\section{FIGURE 3}

Impact of vector control strategies on probability of epidemics (defined as more than three locally acquired cases) and number of locally acquired cases after importation of one case

Vector control after importation confirmation or local confirmation
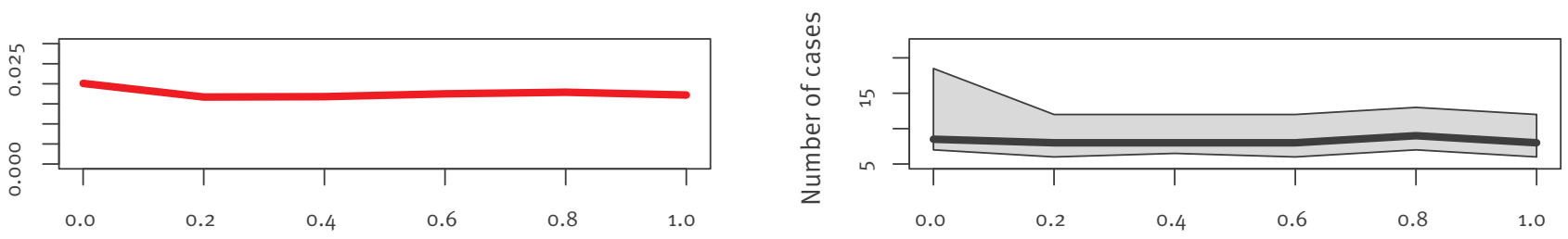

Vector control after importation notification or local confirmation
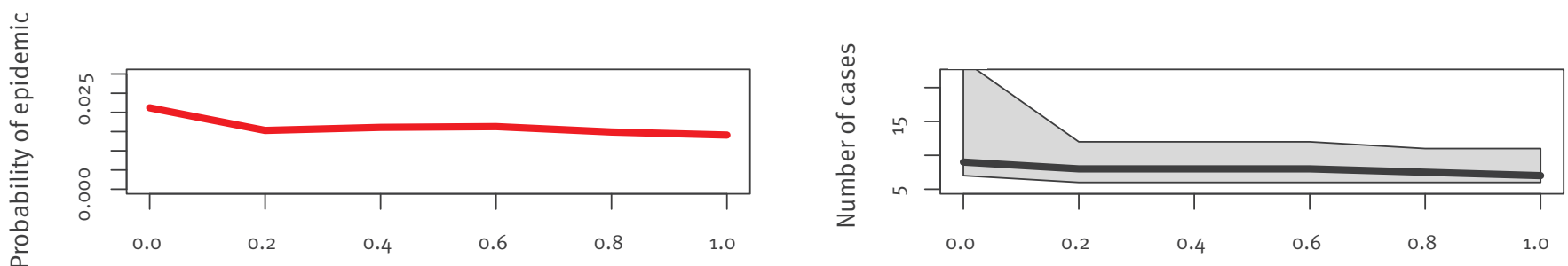

Vector control after importation confirmation or local notification
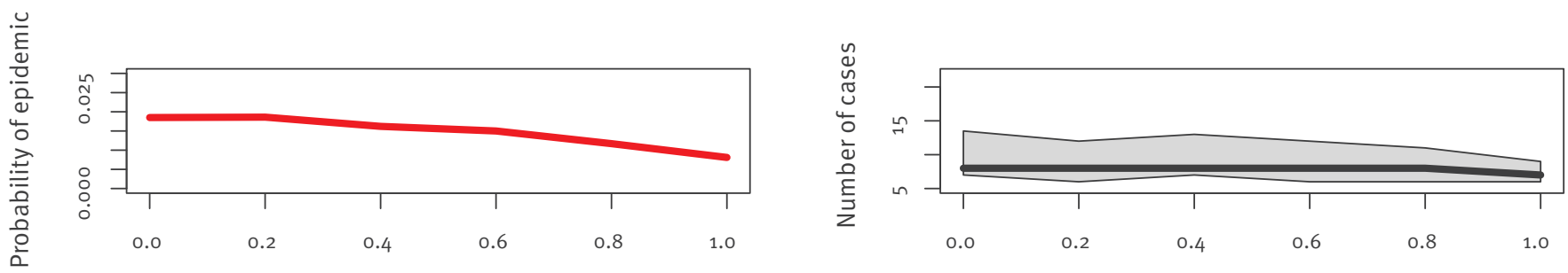

Vector control after importation notification or local notification

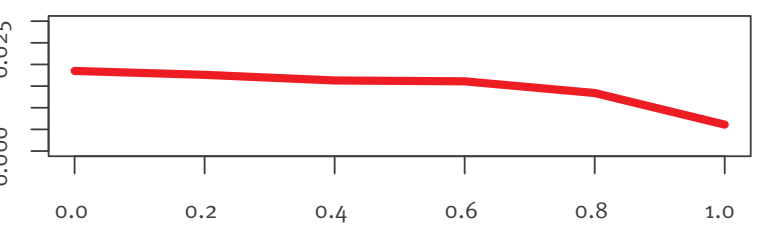

Vector control efficiency

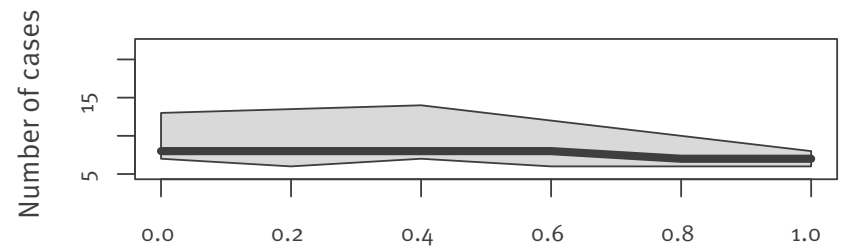

Vector control efficiency

Solid lines represents the median of the simulations.

While starting vector control (VC) after suspicion or confirmation of an imported case did not significantly change the epidemiological outcomes, implementing VC immediately after the notification of a suspected locally acquired case rather than awaiting laboratory confirmation allowed the greatest reduction in the probability of an epidemic. The efficiency of VC represents the percentage of the vector population that was removed by VC operations. 


\section{FIGURE 4}

Costs of diagnostics (left panels) and vector control (right panels) by number of false notifications

Vector control after importation notification or local confirmation

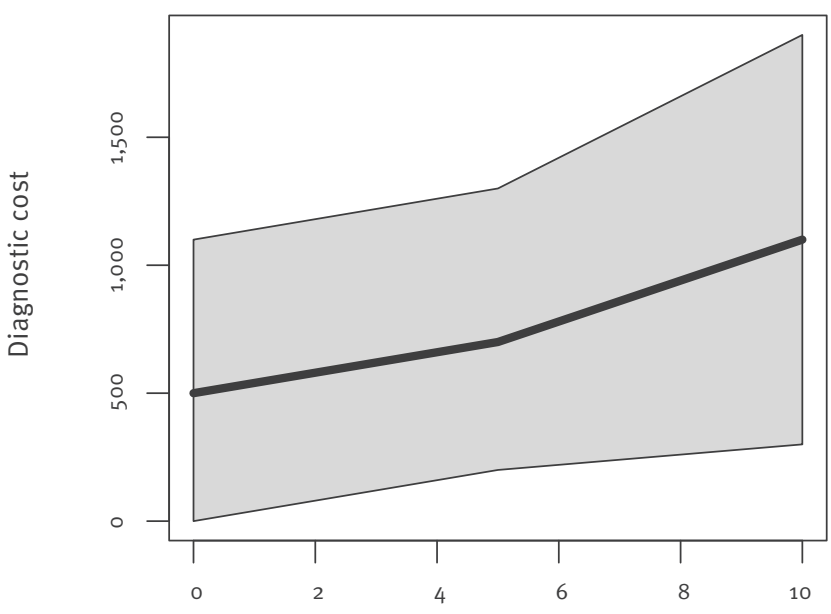

False notifications

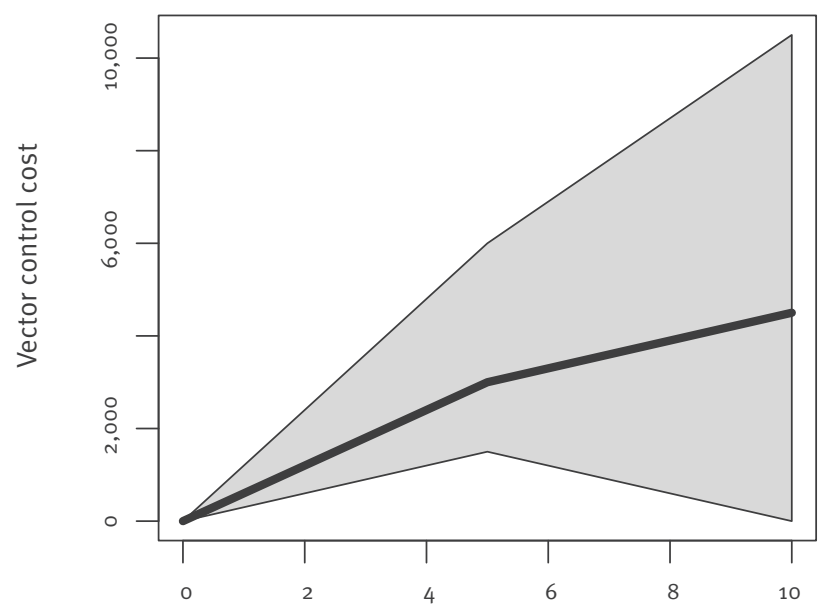

False notifications

Vector control after importation notification or local notification

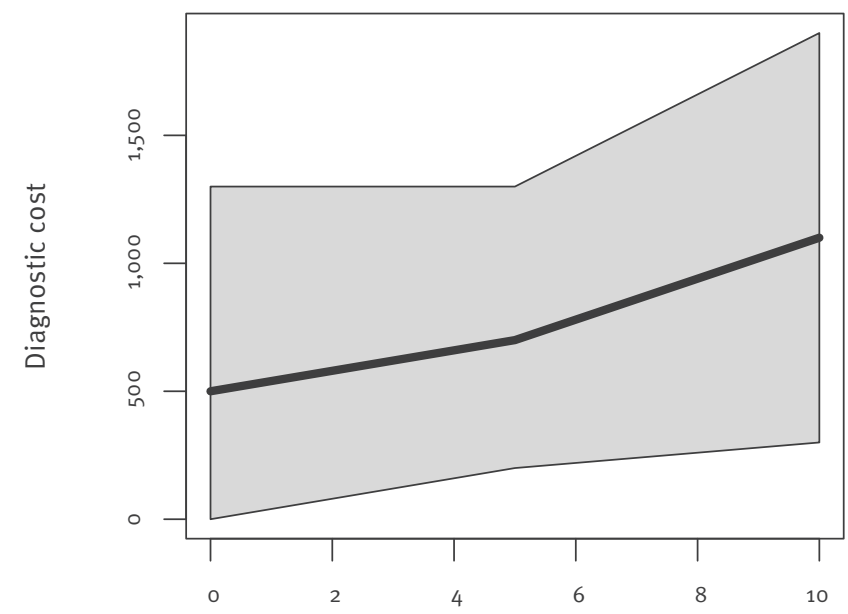

False notifications

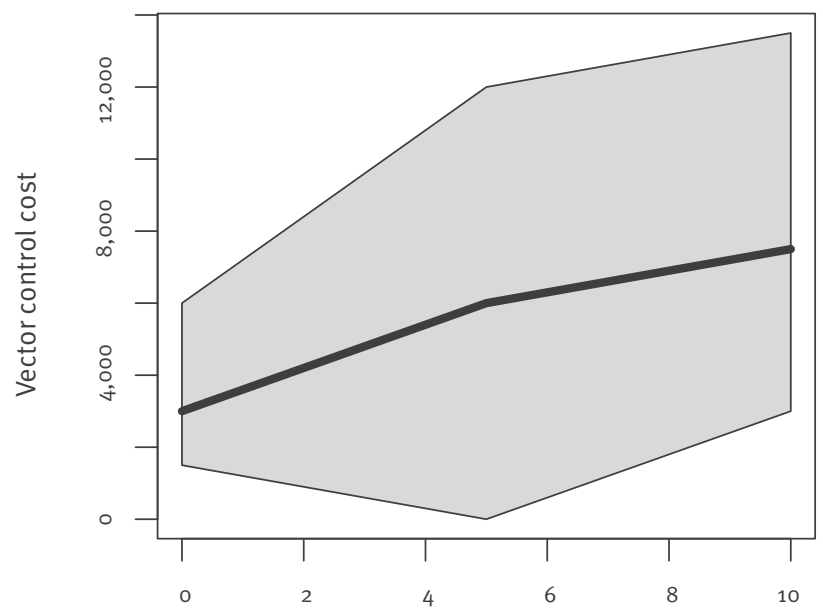

False notifications

Top panels: vector control (VC) triggered after biological confirmation; bottom panels: VC triggered only after suspicion. Costs are in EUR/ha.

become recovered (R) at rate $\sigma$ and cannot be infected again. Similarly, if a susceptible mosquito vector $\left(\mathrm{S}_{\mathrm{m}}\right)$ bites an infectious human $\left(\mathrm{I}_{\mathrm{h}}{ }^{1}, \mathrm{I}_{\mathrm{h}}{ }^{2}\right)$ at rate $a$, the mosquito can become infected $\left(E_{m}\right)$ with the probability $c$ and then infectious $\left(I_{m}\right)$ at the rate $\omega_{m}$, characterised by the inverse of the extrinsic incubation period.

In order to quantify the direct economic costs of false notifications of autochthonous cases to the health system, a third class of individuals $\left(\mathrm{I}_{\mathrm{h}}{ }^{3}\right)$ was considered which represented non-infected individuals who appear symptomatic. These erroneous suspicions may trigger VC if laboratory diagnostic is not required a priori. Furthermore, laboratory tests may return falsepositive results, resulting in erroneous confirmation and autochthonous case notification, triggering VC implementation.

A sub-model considering specifically the case detection and diagnosis process was added on top of this epidemiological framework. When a new individual is added (through importation or local contamination) to 
TABLE

Parameters used in the model assessing public health strategies again chikungunya virus transmission, mainland France

\begin{tabular}{|c|c|c|c|}
\hline Parameters & Description & Values & Source \\
\hline \multicolumn{4}{|c|}{ Diagnostics and vector control } \\
\hline $\mathrm{p}_{\mathrm{i}}$ & Proportion of each diagnostic method & $0.29 ; 0.29 ; 0.42$ & INVS $^{a}$ \\
\hline$p_{i}^{3}$ & Proportion of false positives for each method & $0.027 ; 0.05 ; 0.05$ & INVS $^{\mathrm{a}}$ \\
\hline $1-p_{i}^{1}$ & Proportion of false negatives for each method & $0.03 ; 0.03 ; 0.03$ & INVS $^{a}$ \\
\hline$\varepsilon_{\mathrm{i}}$ & Confirmation rate for each method & $\begin{array}{l}\text { 6; 7; } 14 \text { days per } \\
\text { individual }\end{array}$ & INVS $^{a}$ \\
\hline \multicolumn{2}{|c|}{ Cost for each diagnostic } & EUR 200; 100; 100 & Arbitrary values $^{\mathrm{b}}$ \\
\hline \multicolumn{2}{|c|}{ Cost of Vector control } & EUR 1,500 & Arbitrary value ${ }^{\mathrm{b}}$ \\
\hline \multicolumn{4}{|c|}{ Pathogen } \\
\hline$\sigma$ & Recovery rate & 4 days per individual & {$[20]$} \\
\hline $\mathrm{a}$ & Biting rate of vector species & $\begin{array}{c}0.25 \text { days per } \\
\text { individual } \\
\end{array}$ & $\begin{array}{l}\text { Arbitrary value (no study exists today on } \\
\text { Aedes albopictus in southern France) }\end{array}$ \\
\hline$\mu_{\mathrm{m}}$ & Vector mortality rate & $\begin{array}{c}19 \text { days per } \\
\text { individual }\end{array}$ & [27] \\
\hline b & $\begin{array}{l}\text { Infection probability of a susceptible human } \\
\text { exposed to the pathogen }\end{array}$ & 0.3 & Arbitrary value $^{b}$ \\
\hline c & $\begin{array}{l}\text { Infection probability of a susceptible mosquito } \\
\text { exposed to the pathogen }\end{array}$ & 0.3 & $\begin{array}{l}\text { Arbitrary value based on a virus poorly } \\
\text { adapted to the local mosquito ([22] suggests } \\
\text { o.6 in a well-adapted environment) }\end{array}$ \\
\hline$\theta$ & Efficiency of vector control & Variable & $\begin{array}{c}\text { Variable } \\
\end{array}$ \\
\hline$\omega_{\mathrm{h}}$ & Incubation rate in the human host & 3 days per individual & {$[22]$} \\
\hline$\Omega_{\mathrm{m}}$ & Incubation rate in the mosquito & 2days perindividual & [22] \\
\hline$\Delta$ & Physician consultation rate when infectious & 6days per individual & INVS $^{\mathrm{a}}$ \\
\hline $\mathrm{p}_{\mathrm{A}}$ & $\begin{array}{l}\text { Proportion of locally acquired cases not detected } \\
\text { (including asymptomatic) }\end{array}$ & 0 & Arbitrary value ${ }^{b}$ \\
\hline
\end{tabular}

INVS: Institut de Veille Sanitaire (French Institute for Health Surveillance).

a Values from INVS cover the whole 2014 season in France and have been averaged over this season.

${ }^{\mathrm{b}}$ All arbitrary values have been decided in consultation with the Centre National d'Expertise des Vecteurs.

the number of individuals in class $\mathrm{I}_{\mathrm{h}}{ }^{1}$ (truly infected) or in class $\mathrm{I}_{\mathrm{h}}{ }^{3}$ (wrong suspicion; individual not infected with chikungunya virus), the number of individuals ready for laboratory tests (class $D_{i}^{1}$ or $D_{i}^{3}$ ) will increase at a rate $\Delta_{i}$, where $\Delta_{i}$ represents the notification period of the diagnostic method $i$. The diagnostic methods considered here are PCR confirmation and serology. Each diagnosis is executed at rate $\varepsilon_{i}$ (representing diagnostic time) and the suspected cases can become confirmed $\left(\mathrm{P}_{i}^{j}\right)$ or reversed $\left(\mathrm{N}_{i}^{j}\right)$ with the probability $\mathrm{p}_{i}^{j}$ or $\left(1-\mathrm{p}_{\mathrm{i}}^{\mathrm{j}}\right)$ respectively. For the diagnostic method $i$, $\left(1-p_{i}^{j}\right)$ represents the proportion of false negatives when $\mathrm{j}=1$ (real infectious individuals), while $p_{i}^{j}$ represents the proportion of false positives when $j=3$ (false notification). Finally, each diagnostic method has an associated cost in order to estimate the total direct cost for biological confirmation.

Finally, VC can be implemented when $D_{i}^{j}$ increases by 1 (i.e. when a new infected individual is detected) if the strategy is to implement VC after suspicion $(j=1$ represents real infection while $j=3$ represents erroneous notification). If the strategy is to await laboratory confirmation for both locally acquired and imported cases, VC will be implemented when $\mathrm{P}_{\mathrm{i}}^{\mathrm{j}}$ increases. Tracking the changes allows us to address the four possible strategies, namely implementing VC after clinical suspicion and/or after laboratory confirmation for autochthonous and/or imported cases. VC is based on an integrated approach (source reduction, social mobilisation for destruction of breeding sites such as stagnant water, as well as ultra-low volume (ULV) and thermal fogging for control of adult mosquitoes). Based on experience with mosquito control operators and the biology of $A$. albopictus in temperate areas, we considered that the mosquito population decreases by $\theta \%$, representing the efficacy of VC, during 10 days. After this period, the mosquito population recovers its previous level. Finally, we assumed that a VC operation has an average cost and that the total VC cost is the sum of all VC operations implemented multiplied with this average cost. Details on all transition events can be obtained from the corresponding author and are also available at https://sites.google.com/site/rocheben/ sochakiEtAlAppendix_V3.docx*.

For each studied scenario, we replicated 10,000 simulations through the Gillespie's direct method [18]. In order to have a mosquito population that could be fully reached by VC, we considered a very fine spatial scale 
of one hectare. Similarly, we simulated one single season (six months) because we were focussing here on the short-term impact of the VC strategies. Over this single season, the number of imported cases, which could be of different infectious status, were assumed to arrive uniformly in the population (e.g. one imported case at the beginning, one imported case in the middle and one at the end of the season, if we consider three imported cases). We considered two specific situations for the vector density (ranging from 20 to 800 females per hectare [19]), and two human densities corresponding to the cities of Montpellier and Lyon (46 and 102 inhabitants per hectare, respectively) which are both exposed to increasing vector abundance. All parameters are detailed in the Table and are estimated at the spatial scale of one hectare. Finally, throughout the manuscript, we focus on the probability of the establishment of a local transmission chain (defined as the occurrence of more than five autochthonous cases), called the probability of epidemic, and on the number of autochthonous cases during an outbreak.

\section{Results}

Based on this model, we could derive the basic reproductive number $\left(R_{0}\right)$ that quantifies the number of secondary infections that arise when a single infective host is introduced in a fully susceptible host population. Based on previous results from a chikungunya outbreak in Italy [20], we characterised the $R_{0}$ as:

\section{Formula 1}

$$
R_{0}=\frac{N_{V}}{N_{h}} \frac{b c}{\sigma \mu_{m}} \frac{\omega_{m}}{\omega_{m}+\mu_{m}}
$$

Based on the parameters detailed in the Table, we consider in this study $R_{0}$ values ranging from 0.16 to 6.72 in Montpellier and from 0.07 to 2.99 in Lyon (according to vector and human densities), which is consistent with current estimations [20].

In order to quantify the net epidemiological impact of imported infectious individuals, we first considered a simple case without $\mathrm{VC}$ but with perfect tracking of infectious individuals (no asymptomatic cases nor false notification). As could be expected, we found that an increasing vector density significantly increased the probability of an epidemic (Figure 2), from o\% to $2 \%$, and therefore the size of the epidemic number of locally acquired cases). However, the number of imported cases had a very weak impact on the probability of an epidemic, from o\% to $0.1 \%$ for low vector density, yielding a large confidence interval for the size of the ensuing epidemic because the smaller number of simulations on which size of the epidemic could be calculated.

Based on the high vector density in Lyon (with a $R_{0}$ of 2.99), we then focused on the four possible strategies that could be implemented, namely implementing VC (i) after notification of clinical suspicion of imported case(s) and laboratory confirmation of autochthonous transmission, (ii) after laboratory confirmation for either imported or autochthonous cases, (iii) after laboratory confirmation of an imported case or after clinical suspicion of an autochthonous case without laboratory confirmation or (iv) after a clinically suspected case, either imported or autochthonous. VC efficacy had a considerable influence on epidemiological outcomes, although the impact was stronger on the probability than on the size of epidemics (Figure 3).

Our model shows that not all strategies are equivalent. While the size of the epidemic remained roughly similar across the strategies, the probability of an epidemic was reduced when VC was started as soon as autochthonous transmission was suspected, without awaiting laboratory confirmation of cases. Starting VC upon suspicion or after confirmation of imported cases did not alter the epidemiological outcome.

Assuming a VC efficacy of $80 \%$ and one imported case within the area, we focused on direct economic costs of the two strategies for autochthonous cases. For this, we introduced non-infectious individuals $\left(\mathrm{I}^{3}\right)$ who nonetheless were entered in the surveillance system through erroneous suspicion and tested for laboratory confirmation (with the possibility of false positives). Figure 4 shows that the cost of VC increased significantly when VC was implemented after suspicion of cases rather than after their confirmation. However, the impact on diagnostic cost was weak.

\section{Discussion}

This first theoretical exploration chiefly aimed to highlight the various factors influencing the probability of a chikungunya epidemic and its spread in southern France and to show the possible consequences of different public health strategies. In this work, we considered four strategies: (i) VC after suspicion of an imported or autochthonous case, (ii) VC after suspicion of an imported case or after confirmation of a locally acquired case, (iii) VC after confirmation of an imported case or suspicion of autochthonous transmission or (iv) VC after confirmation of an imported or locally acquired case. We found that implementing VC immediately after notification of a suspected case did not significantly change the size of the epidemic, compared with delaying implementation of VC until laboratory confirmation. In other words, awaiting confirmation before implementing VC is not expected to result in a larger epidemic (with the current notification delay). However, our results suggest that awaiting laboratory confirmation before implementing VC around autochthonous cases may result in higher probability of an epidemic. However, the optimal strategy is also subject to economically constraints. In our model, implementing VC readily after suspicion was more expensive than after laboratory confirmation because of erroneous suspicions. Moreover, repeated VC operations are generally not feasible in the field for logistical 
reasons and concerns of the population about repeated insecticide spraying.

The fact that the different strategies for imported cases did not significantly change the probability of an epidemic or its size, was mainly due to the delay between the infectious individuals entering the surveillance system and the notification of these imported cases. In 2014, this delay was six days on average, i.e. when the infectious individual has almost finished their infectious period and had potentially transmitted the virus to local Aedes mosquitoes. In addition, we observed that this delay had to be decreased significantly if we aimed to decrease significantly probability of epidemic following the introduction of imported cases (data not shown; details can be obtained from the corresponding author and are also available at https://sites.google. com/site/rocheben/sochakiEtAlAppendix_V3.docx*).

Some assumptions made by this model deserve to be discussed. Firstly, some epidemiological parameters were unknown and were fixed arbitrarily. This is especially relevant for the probability that a susceptible vector gets infected after biting an infectious human (parameter $c$ ). Indeed, the vector competence of $A e$. albopictus populations in Metropolitan France will depend on the chikungunya strain infecting the human population and therefore on the geographic origin of the imported case. Nevertheless, we have conducted a sensitivity analysis (details can be obtained from the corresponding author and are also available at https:// sites.google.com/site/rocheben/sochakiEtAlAppendix_V3.docx ${ }^{*}$ ), in which our main conclusions remained valid for a large range of parameters. Secondly, our estimated costs, for both diagnostics and VC, allowed to compare between the different scenarios but they underestimated the real costs since only direct costs were included. Finally, we considered here a very fine spatial scale (one hectare). As a consequence, we neglected the important component of human movements. The situation described here corresponds to outbreaks very localised in space, for which this scale is relevant. Nevertheless, in the case of a more diffuse outbreak, this model would need to be extended to a broader scale.

An important result arising from our paper is the influence of vector density on both probability and size of epidemics. While this result could have been expected based on the existing literature $[17,21]$, it is nevertheless worth pointing out that such a relationship is strongly dependent on how the transmission force between vector and hosts is modelled. Here, we used the most popular way of vector-borne disease modelling (asymmetric frequency-dependent transmission). However, we could also show that another way of modelling this transmission force, through a symmetric frequency-dependent process, can result in the absence of a relationship between vector density and size of the epidemic (data not shown; details can be obtained from the corresponding author and are also available at https://sites.google.com/site/rocheben/ sochakiEtAlAppendix_V3.docx*), as empirical data suggest for dengue [22]. Nevertheless, the only qualitative change arising with this other kind of transmission force is the influence of vector density. Therefore, although we cannot draw a definite conclusion regarding the influence of vector density, the main conclusion (implementing VC after suspicion for autochthonous cases is the best way to reduce the consequences of an epidemic) remains valid.

Other modelling studies have focused on chikungunya virus transmission in other epidemiological settings $[20,23,24]$, some with a focus on VC [25]. Some studies have highlighted that certain areas are more suitable than others for chikungunya epidemics [26]. Nevertheless, this study is as far as we know, the first to focus on such concerns (large number of imported cases combined with local presence of vector) in Europe. While specifically designed the model to mimic the French surveillance system, we believe that it can easily be adapted to other surveillance systems.

It is worth pointing out that the outcomes of our model matched the size of the epidemic observed in Montpellier in 2014 [15] where 11 autochthonous cases were recorded after the introduction of a single imported case. While such agreement cannot validate the whole framework on its own, it nevertheless underlines that the approach is worth exploring and may provide useful insights for public health strategy.

The current strategy in France foresees the implementation of VC after suspicion of imported cases or after confirmation of autochthonous cases. Nevertheless, when local transmission is proven, current strategy assumes that VC is applied around every suspected autochthonous case without awaiting laboratory confirmation. This strategy is used because imported cases are relatively easy to discern (travel in an endemic area) and the positive predictive value of the clinical case definition is higher in this high-prevalence population group. Conversely, the low predictive power of the clinical case definitions in the resident human population that has not travelled to endemic areas leads to reporting of many patients not infected by chikungunya virus, creating high costs for unnecessary interventions. Overall, our theoretical framework suggests that such a strategy, in the current conditions (and especially with the current delay in case reporting), could be the most efficient one, both in economic and epidemiological terms, if proof of local transmission can be established rapidly enough. The model developed here aimed to be as generic as possible, so that it can be used as a general framework to test other scenarios or to analyse VC strategies for other diseases.

\section{${ }^{\star}$ Note}

Supplementary information made available by the authors on an independent website is not edited by Eurosurveillance, 
and Eurosurveillance is not responsible for the content. The material can be accessed at: https://sites.google.com/site/ rocheben/sochakiEtAlAppendix_V3.docx.

\section{Acknowledgements}

We thank the ANRS for funding and the Direction Generale de la Santé for their insights into surveillance system.

\section{Conflict of interest}

None declared.

\section{Authors' contributions}

$B R, D F, F S$ and MCP have designed the study. TS and BR did the modelling analysis. FJ and YP have improved the entomological component of the model. HN, MCP AS improved the surveillance component of the model and analysed the surveillance data during the 2014 season. All authors have significantly contributed the writing of the manuscript.

\section{References}

1. Daszak P, Cunningham AA, Hyatt AD. Emerging infectious diseases of wildlife--threats to biodiversity and human health.Science. 2000;287(5452):443-9. DOI: 10.1126/ science.287.5452.443 PMID: 10642539

2. McMichael AJ. Environmental and social influences on emerging infectious diseases: past, present and future.Philos Trans R Soc Lond B Biol Sci. 2004;359(1447):1049-58. DOI: 10.1098/rstb.2004.1480 PMID: 15306389

3. Altizer S, Ostfeld RS, Johnson PTJ, Kutz S, Harvell CD. Climate change and infectious diseases: from evidence to a predictive framework.Science. 2013;341(6145):514-9. DOI: 10.1126/ science.1239401 PMID: 23908230

4. Keesing F, Belden LK, Daszak P, Dobson A, Harvell CD, Holt RD, et al. Impacts of biodiversity on the emergence and transmission of infectious diseases. Nature. 2010;468(7324):647-52. DOI: 10.1038/nature09575 PMID: 21124449

5. Bradley CA, Altizer S. Urbanization and the ecology of wildlife diseases.Trends Ecol Evol. 2007;22(2):95-102. DOI: 10.1016/j. tree.2006.11.001 PMID: 17113678

6. Benedict MQ, Levine RS, Hawley WA, Lounibos LP. Spread of the tiger: global risk of invasion by the mosquito Aedes albopictus.Vector Borne Zoonotic Dis. 2007;7(1):76-85. DOI: 10.1089/vbz.2006.0562 PMID: 17417960

7. Chevillon C, Briant L, Renaud F, Devaux C. The Chikungunya threat: an ecological and evolutionary perspective.Trends Microbiol. 2008;16(2):80-8. DOI: 10.1016/j.tim.2007.12.003 PMID: 18191569

8. Rezza G, Nicoletti L, Angelini R, Romi R, Finarelli AC, Panning $M$, et al. Infection with chikungunya virus in Italy: an outbreak in a temperate region. Lancet. 2007;370(9602):1840-6. DOI: 10.1016/So140-6736(07)61779-6 PMID: 18061059

9. Rezza G. Aedes albopictus and the reemergence of Dengue. BMC Public Health. 2012;12(1):72. DOI: 10.1186/1471-2458-1272 PMID: 22272602

10. Nasci RS. Movement of chikungunya virus into the Western hemisphere.Emerg Infect Dis. 2014;20(8):1394-5. DOI: 10.3201/ eid2008.140333 PMID: 25061832

11. Van Bortel W, Dorleans F, Rosine J, Blateau A, Rousset D, Matheus S, et al. Chikungunya outbreak in the Caribbean region, December 2013 to March 2014, and the significance for Europe. Euro Surveill. 2014;19(13):20759. DOI: 10.2807/15607917.ES2014.19.13.20759 PMID: 24721539

12. Cauchemez S, Ledrans M, Poletto C, Quenel P, de Valk H, Colizza V, et al. Local and regional spread of chikungunya fever in the Americas. Euro Surveill. 2014;19(28):20854. DOI: 10.2807/1560-7917.ES2014.19.28.20854 PMID: 25060573

13. Mansuy JM, Grouteau E, Mengelle C, Claudet I, Izopet J. Chikungunya in the Caribbean--threat for Europe.Emerg Infect Dis. 2014;20(8):1423-5. DOI: 10.3201/eid2008.140650 PMID: 25062125

14. Roche B, Léger L, L'Ambert G, Lacour G, Foussadier R, Besnard $G$, et al. The spread of Aedes albopictus in Metropolitan
France: contribution of environmental drivers and human activities and predictions for a near future. PLoS One. 2015;10(5):e0125600. DOI: 10.1371/journal.pone.0125600 PMID: 25962160

15. Paty MC, Six C, Charlet F, Heuzé G, Cochet A, Wiegandt A, et al. Large number of imported chikungunya cases in mainland France, 2014: a challenge for surveillance and response. Euro Surveill. 2014;19(28):20856. DOI: 10.2807/1560-7917. ES2014.19.28.20856 PMID: 25060572

16. Chikungunya et dengue - Données de la surveillance renforcée en 2015. [Chikungunya and dengue - enhanced surveillance data 2015]. Paris: Institut de Veille Sanitaire; 2015. French. Available from: http://www.invs.sante.fr/ Dossiers-thematiques/Maladies-infectieuses/Maladies-atransmission-vectorielle/Dengue/Donnees-epidemiologiques/ France-metropolitaine/Chikungunya-et-dengue-Donnees-de-lasurveillance-renforcee-en-2014

17. Keeling MJ, Rohani P. Modeling infectious diseases in humans and animals. Princeton University Press; 2008.

18. Gillespie DT. Approximate accelerated stochastic simulation of chemically reacting systems.J Chem Phys. 2001;115(4):1716-33. DOI: $10.1063 / 1.1378322$

19. Cianci D, Van den Broek J, Caputo B, Marini F, Della Torre A, Heesterbeek $\mathrm{H}$, et al. Estimating mosquito population size from mark-release-recapture data. J Med Entomol. 2013;50(3):533-42. DOI: 10.1603/ME12126 PMID: 23802447

20. Poletti P, Messeri G, Ajelli M, Vallorani R, Rizzo C, Merler S. Transmission potential of chikungunya virus and control measures: the case of Italy.PLoS One. 2011;6(5):e18860. DOI: 10.1371/journal.pone.0018860 PMID: 21559329

21. Roche B, Rohani P, Dobson AP, Guégan JFJ-F. The impact of community organization on vector-borne pathogens. Am Nat. 2013;181(1):1-11. DOI: 10.1086/668591 PMID: 23234841

22. Bowman LR, Runge-Ranzinger S, McCall PJ. Assessing the relationship between vector indices and dengue transmission: a systematic review of the evidence.PLoS Negl Trop Dis. 2014;8(5):e2848. DOI: 10.1371/journal.pntd.0002848 PMID: 24810901

23. Robinson M, Conan A, Duong V, Ly S, Ngan C, Buchy P, et al. A model for a chikungunya outbreak in a rural Cambodian setting: implications for disease control in uninfected areas. PLoS Negl Trop Dis. 2014;8(9):e3120. DOI: 10.1371/journal. pntd.0003120 PMID: 25210729

24. Dumont Y, Chiroleu F, Domerg C. On a temporal model for the Chikungunya disease: modeling, theory and numerics. Math Biosci. 2008;213(1):80-91. DOI: 10.1016/j.mbs.2008.02.008 PMID: 18394655

25. Dumont Y, Chiroleu F. Vector control for the Chikungunya disease.Math Biosci Eng. 2010;7(2):313-45. DOI: 10.3934/ mbe.2010.7.313 PMID: 20462292

26. Dhimal M, Gautam I, Joshi HD, O’Hara RB, Ahrens B, Kuch $U$. Risk factors for the presence of chikungunya and dengue vectors (Aedes aegypti and Aedes albopictus), their altitudinal distribution and climatic determinants of their abundance in central Nepal.PLoS Negl Trop Dis. 2015;9(3):eoo03545. DOI: 10.1371/journal.pntd.0003545 PMID: 25774518

27. Nur Aida H, Abu Hassan A, Nurita AT, Che Salmah MR, Norasmah B. Population analysis of Aedes albopictus (Skuse) (Diptera:Culicidae) under uncontrolled laboratory conditions. Trop Biomed. 2008;25(2):117-25.PMID: 18948882

\section{License and copyright}

This is an open-access article distributed under the terms of the Creative Commons Attribution (CC BY 4.0) Licence. You may share and adapt the material, but must give appropriate credit to the source, provide a link to the licence, and indicate if changes were made.

This article is copyright of the authors, 2016. 